\section{(6) OPEN ACCESS}

\title{
International Cancer of the Pancreas Screening (CAPS) Consortium summit on the management of patients with increased risk for familial pancreatic cancer
}

\author{
Marcia Irene Canto, ${ }^{1}$ Femme Harinck, ${ }^{2}$ Ralph $\mathrm{H}$ Hruban, ${ }^{3}$ George Johan Offerhaus, ${ }^{4}$ \\ Jan-Werner Poley, ${ }^{2}$ Ihab Kamel, ${ }^{5}$ Yung Nio, ${ }^{6}$ Richard S Schulick, ${ }^{7}$ Claudio Bassi, ${ }^{8}$ \\ Irma Kluijt, ${ }^{9}$ Michael J Levy, ${ }^{10}$ Amitabh Chak, ${ }^{11}$ Paul Fockens, ${ }^{12}$ Michael Goggins, ${ }^{1}$ \\ Marco Bruno, ${ }^{2}$ on behalf of the International Cancer of the Pancreas Screening (CAPS) \\ Consortium
}

\begin{abstract}
- Additional data (Appendix) are published online only. To view these files please visit the journal online (http://dx.doi.org/ 10.1136/gutjpl-2012-303108).

For numbered affiliations see end of article

\section{Correspondence to Dr Marcia Irene Canto, Division of Gastroenterology, Johns Hopkins University, The Sol Goldman Pancreatic Cancer Research Center, $1830 \mathrm{E}$ Monument Street, Room 427, Baltimore, MD 21205, USA: mcanto@jhmi.edu}

Received 19 June 2012 Accepted 17 September 2012 Published Online First 7 November 2012

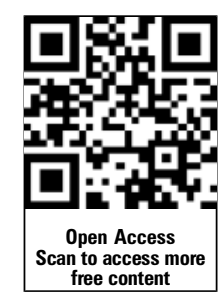

\section{ABSTRACT}

Background Screening individuals at increased risk for pancreatic cancer (PC) detects early, potentially curable, pancreatic neoplasia.

Objective To develop consortium statements on screening, surveillance and management of high-risk individuals with an inherited predisposition to PC. Methods A 49-expert multidisciplinary international consortium met to discuss pancreatic screening and vote on statements. Consensus was considered reached if $\geq 75 \%$ agreed or disagreed.

Results There was excellent agreement that, to be successful, a screening programme should detect and treat T1N0M0 margin-negative PC and high-grade dysplastic precursor lesions (pancreatic intraepithelial neoplasia and intraductal papillary mucinous neoplasm). It was agreed that the following were candidates for screening: first-degree relatives (FDRs) of patients with PC from a familial PC kindred with at least two affected FDRs; patients with Peutz-Jeghers syndrome; and p16, $B R C A 2$ and hereditary non-polyposis colorectal cancer (HNPCC) mutation carriers with $\geq 1$ affected FDR. Consensus was not reached for the age to initiate screening or stop surveillance. It was agreed that initial screening should include endoscopic ultrasonography (EUS) and/or MRI/magnetic resonance cholangiopancreatography not CT or endoscopic retrograde cholangiopancreatography. There was no consensus on the need for EUS fine-needle aspiration to evaluate cysts. There was disagreement on optimal screening modalities and intervals for follow-up imaging. When surgery is recommended it should be performed at a high-volume centre. There was great disagreement as to which screening abnormalities were of sufficient concern to for surgery to be recommended.

Conclusions Screening is recommended for high-risk individuals, but more evidence is needed, particularly for how to manage patients with detected lesions. Screening and subsequent management should take place at high-volume centres with multidisciplinary teams, preferably within research protocols.

\section{INTRODUCTION}

Pancreatic ductal adenocarcinoma (PC) is a deadly disease. It remains the fourth most common cause of death from cancer in the USA ${ }^{1}$ and one of the deadliest cancers in the world. Although treatments have improved, average PC 5-year survival is $<5 \%$. Because pancreatic neoplasia detected early is potentially curable, there is interest in pancreatic screening. Because of the low incidence of PC in the general population, population-based screening has not been recommended.

Selective screening of individuals at increased risk for PC (high-risk individuals (HRIs)) based on their family history or identifiable genetic predisposition is considered worthwhile. Over the past decade, centres in the USA and Europe initiated pancreatic screening programmes. Single $-{ }^{2-10}$ and multicentre ${ }^{8} 11$ cohort studies have evaluated the diagnostic yield of screening (detection of asymptomatic precursor lesions and PCs at baseline and follow-up) using different imaging modalities and study populations (table 1).

\section{AIMS AND METHODS \\ Scope and purpose}

The International Cancer of the Pancreas Screening (CAPS) Consortium was formed in 2010 to help organise global pancreatic screening. After prior formulation of key topics for discussion, the CAPS Consortium held a multidisciplinary consensus conference. The statements developed at this conference provide recommendations related to the following questions: (1) Who should be screened? (2) How should HRIs be screened and followed up? (3) When should surgery be performed? (4) What are the goals of screening and what outcome should be considered a success?

\section{Consortium participants}

The conference chairs (Professors Canto and Bruno) selected an international multidisciplinary group of 50 experts from 10 countries in four continents representing the fields of epidemiology, genetics, gastroenterology, radiology, oncology, surgery and pathology. Participant selection was based upon expertise, publications and participation in ongoing PC screening and surveillance programmes. The group included physicians, scientists, nurses and genetic counsellors from community-based practices, academic institutions and cancer centres. 
Table 1 Summary of diagnostic yield of familial pancreatic cancer screening and surveillance programmes

\begin{tabular}{|c|c|c|c|}
\hline Study & High-risk group & Imaging tests & Diagnostic yield*n (\%) \\
\hline Brentnall 1999 (1) $n=14$ & FPC & $\mathrm{EUS}+\mathrm{ERCP}+\mathrm{CT}$ & $7 / 14(50) \dagger$ \\
\hline Kimmey $2002 \mathrm{n}=46 \ddagger$ & FPC & EUS; ERCP§ & $12 / 46(26) \dagger$ \\
\hline Canto 2004 (2) n=38 & FPC, PJS & EUS; ERCP§, EUS-FNA§, CT§ & $2 / 38(5.3) \dagger$ \\
\hline Canto 2006 (3) n=78 & FPC, PJS & EUS; CT§,EUS-FNA§, ERCP§ & $8 / 78(10.3) \pi, \dagger$ \\
\hline Poley 2009 (4) $n=44$ & FPC, BRCA, PJS, p16, p53, HP & EUS;CT§, MRI§ & $10 / 44(23)$ \\
\hline Langer $2009(5) n=76$ & $\mathrm{FPC}, \mathrm{BRCA}$ & EUS+MRCP; EUS-FNA§ & $1 / 76(1.3) \pi, \dagger$ \\
\hline Verna $2010(6) n=51$ & FPC, BRCA, p16 & EUS and/or MRCP & $6 / 51(12) \dagger$ \\
\hline Ludwig $2011 \mathrm{n}=109$ & $\mathrm{FPC}, \mathrm{BRCA}$ & MRCP; EUS§, EUS-FNA§ & $9 / 109(8.3) \uparrow$ \\
\hline Vasen 2011 (7) $n=79$ & $\mathrm{p} 16$ & MRI/MRCP & $14 / 79+(18)$ \\
\hline Al-Sukhni 2011 (8) $n=262$ & FPC, BRCA, PJS, p16, HP & MRI; CT§, EUS§, ERCP§ & 19/262ף (7.3) \\
\hline Schneider $2011(9)^{* *} \mathrm{n}=72$ & FPC, BRCA, PALB2 & EUS+MRCP & $11 / 72(15) \uparrow$ \\
\hline Canto $2012(10) n=216$ & FPC, BRCA, PJS & CT, MRI/MRCP, EUS; ERCP§ & $5 / 216(2.3) \dagger-92 / 216$ (43) \\
\hline
\end{tabular}

${ }^{*}$ Yield is defined as the detection of any pathologically proven (pre)malignant lesion ( $\geq$ PanIN-2/IPMN and pancreatic adenocarcinoma) and lesions that are morphologically suspicious for branch-duct IPMNs.

tIncludes only pathologically proven pancreatic neoplasms (histology or cytology).

‡Continuation of Brentnall 1999, included 14 high-risk individuals from Brentnall 1999.

$\S$ Test performed only as an additional test for detected abnormalities.

TIncludes baseline and follow-up.

${ }^{* *}$ Continuation of Langer 2009, includes high-risk individuals from this series.

ERCP, endoscopic retrograde cholangiopancreatography; EUS, endoscopic ultrasonography; FNA, fine-needle aspiration; FPC, familial pancreatic cancer; IPMN, intraductal papillary mucinous neoplasm; MRCP, magnetic resonance cholangiopancreatography; PJS, Peutz-Jeghers syndrome; PanIN, pancreatic intraepithelial neoplasia.

\section{Conference proceedings}

The international CAPS Consortium held a 2-day conference in February 2011, in Baltimore, Maryland, USA. A comprehensive literature search was performed and relevant publications were reviewed by conference chairs and representative experts. At the meeting, experts outlined the current state of the field. Thereafter, workgroups comprising geneticists/epidemiologists, gastroenterologists, radiologists, surgeons and pathologists met to discuss topics relevant to each specialty. Multidisciplinary groups met in breakout sessions to formulate concise statements for voting (see table 1, online Appendix). Gaps in knowledge and areas of disagreement and agreement were also identified and specifically discussed. The statements were presented after plenary and workgroup discussions, and anonymous voting was performed using touchpad technology at the end of the meeting.

The resulting 100 consensus statements were reviewed and refined after the meeting and then voted on by 49 of 50 participants by anonymous electronic survey. Participants voted on multiple choice questions on a five-point scale (eg, a =definitely agree, $b=$ moderately agree, $c=$ neutral, $d=$ moderately disagree, $e=$ definitely disagree) or five-item selection list. A statement was accepted if $\geq 75 \%$ of the participants voted 'agree' or 'disagree'. Statements that did not reach consensus are listed separately (Appendix 1).

The Appraisal of Guidelines Research and Evaluation process for assessment of quality of evidence and strength of recommendations $^{1213}$ was used to determine if the available literature was sufficient to make and grade recommendations. Evidence was graded based upon study design (randomised controlled trial=high, observational study=low, any other evidence= $=$ very low), study quality, consistency and directness of evidence. ${ }^{12}$ The grade of evidence was modified if there was strong evidence of association (relative risk $(R R)>2$ ) (eg, consistent evidence from multiple observational studies, or evidence of a dose-response gradient). ${ }^{12}$ The strength of the group's recommendation statement was based upon Grading of Recommendations Assessment, Development and Evaluation (GRADE) definitions for quality improvement and guidelines development ${ }^{12}{ }^{14}$ : 1 (strong)= 'definitely do it', 2 (weak)='probably do it', 3 (no recommendation), 4 (weak)= 'probably don't do it' and 5 (strong) $=$ 'definitely don't do it'.

\section{RECOMMENDATION STATEMENTS}

Each statement includes its grade of evidence, the voting results (table 2) and a brief discussion.

\section{Who should be screened?}

Since the incidence of PC in the general population is low (lifetime risk $1.3 \%$ ), screening is not recommended for the general population, but instead for individuals considered to be at high risk of developing the disease (ie, $>5 \%$ lifetime risk, or fivefold increased RR). The main tool used to quantify PC risk is still the family history; risk stratification is determined from the number of affected family members and the relationships among at-risk individuals. ${ }^{15}$ Gene testing can identify a family's underlying genetic susceptibility, but it has a limited role because the genetic basis of much of the inherited susceptibility to PC remains unexplained. Additional PC susceptibility genes may be discovered in the near future that should improve our ability to identify individuals who would benefit most from pancreatic screening.

\section{Patients with a family history of PC}

Individuals with three or more blood relatives with PC, with at least one affected first-degree relative (FDR,) should be considered for screening (agree $91.9 \%$, grade moderate, 'probably do it'). Those with at least two affected FDRs should be considered for screening (agree 91.9\%, grade moderate, 'probably do it'). Individuals with two affected blood relatives with PC, with at least one FDR, should be considered for screening (agree 77.5\%, grade low, 'probably do it').

These recommendations for screening are primarily based on evidence of increased risk, rather than a proven efficacy of screening. Prospective studies demonstrate an increased risk of developing PC in unaffected FDRs that depends on the number of relatives with PC. ${ }^{16}$ This risk has been estimated to be 6.4-fold greater in individuals with two FDRs with PC (lifetime risk $8-12 \%{ }^{17}$ ) and 32 -fold greater in individuals with three or more FDRs with PC (lifetime risk $40 \%{ }^{17}$ ). Among kindreds with familial PC, risk is higher in kindreds with a young-onset PC (age <50 years, $R R=9.3$ ) compared with kindreds 
Table 2 Summary of consensus statements for the management of high risk individuals

Who should be screened?

Statements

A1 Individuals with three or more affected blood relatives, with at least one affected FDR, should be considered for screening.

A2 Individuals with at least two affected FDRs with PC, with at least one affected FDR, should be considered for screening once they reach a certain age.

A3 Individuals with two or more affected blood relatives with PC, with at least one affected FDR, should be considered for screening.

A4 All patients with Peutz-Jeghers syndrome should be screened, regardless of family history of PC.

A5 $\quad$ 16 carriers with one affected FDR should be considered for screening.

A6 BRCA2 mutation carriers with one affected FDR should be considered for screening.

A7 BRCA2 mutation carriers with two affected family members (no FDR) with PC should be considered for screening.

A8 PALB2 mutation carriers with one affected FDR should be considered for screening.

A9 Mismatch repair gene mutation carriers (Lynch syndrome) with one affected FDR should be considered for screening.

How should high-risk individuals be screened?

Statements

B1 Initial screening should include (multiple answers allowed):

EUS $83.7 \%$, MRI/MRCP $73.5 \%$, CT $26.5 \%$, abdominal ultrasound $14.3 \%$, ERCP $2.0 \%$.

B2 When previous screening did not detect an abnormality that met criteria for shortening of the interval or surgical resection, follow-up screening should include (multiple answers allowed): EUS 79.6\% MRI/MRCP $69.4 \%$, CT 22.4\%, abdominal ultrasound $4.1 \%$, ERCP $2.0 \%$.

B3 Standardised nomenclature should be used to define chronic pancreatitis-like abnormalities.

B4 Whenever a cystic lesion is detected, an additional ERCP should not be performed.

B5 Patients with a cystic lesion without worrisome features for malignancy should have an imaging test after 6-12 months.

B6 When a solid lesion is detected, CT should also be performed.

B7 When a solid lesion is detected, ERCP should not be performed.

B8 When a solid lesion is detected at baseline with an indeterminate diagnosis and the patient is not referred for immediate surgery, imaging should be repeated after 3 months.

B9 When a new solid lesion is detected at follow-up with an indeterminate diagnosis and the patient is not referred for immediate surgery, imaging should be repeated after 3 months.

B10 If an indeterminate main pancreatic duct stricture without a mass is detected, repeat imaging should be performed within 3 months. When should surgery be performed?

Statements

C1 Screening should only be offered to individuals who are candidates for surgery.

C2 Pancreatic resections should be performed at specialty centres (taking into account volume, morbidity and mortality rates and expertise available).

C3 Intraoperatively, further pancreatectomy (up to a possible total) should be performed in patients with otherwise reasonable life expectancy to achieve R0 resection of cancer.

C4 Intraoperatively, further pancreatectomy (up to a possible total) should not be performed in a patient with otherwise reasonable life expectancy and no cancer but with unifocal PanIN-2 in the resected specimen but not at the margin.

C5 Postoperatively, further pancreatectomy (up to a possible total) should be not performed in patients with otherwise reasonable life expectancy in a patient without cancer in the resected specimen but with PanIN-2 at margin.

C6 Postoperatively, further pancreatectomy (up to possible total) should be not be performed in patients with otherwise reasonable life expectancy in a patient who did not have cancer but had unifocal PanIN-2 in the resected specimens but not at the margin.

C7 Postoperatively, further pancreatectomy (up to a possible total) should be not performed in patients with otherwise reasonable life expectancy in a patient without cancer but who has multifocal PanIN-2 in the resected specimens but not at the margin.

What are the goals of screening? What outcome(s) would be considered a 'success'?

Statements

D1 Resectable carcinoma is a potential target for early detection and treatment.

D2 PanINs are a potential target for early detection and treatment.

D3 IPMNs are a potential target for early detection and treatment.

D4 Detection and treatment of multifocal PanIN-3 should be considered a success of a screening/surveillance programme.

D5 Detection and treatment of IPMNs with high-grade dysplasia should be considered a success of a screening/surveillance programme.

D6 Detection and treatment of invasive cancer-T1N0M0 detected at baseline should be considered a success of a screening programme.

D7 Treatment of invasive cancer-T1NOMO detected at follow-up should be considered a success of a screening programme.

D8 Detection and treatment of invasive cancer $>$ T1NOMO resectable with margins negative at baseline, should be considered a success of a screening programme.

ERCP, endoscopic retrograde cholangiopancreatography; EUS, endoscopic ultrasonography; FDR, first-degree relative; IPMN, intraductal papillary mucinous neoplasm; MRCP, magnetic resonance cholangiopancreatography; PC, pancreatic cancer; PanIN, pancreatic intraepithelial neoplasia.

without. ${ }^{18}$ No consensus was reached on whether to screen individuals without an affected FDR, including individuals with a young-onset PC relative, or patients with new-onset diabetes (Appendix).

\section{Mutation carriers}

Germline mutations in the BRCA2, PALB2, p16, STK11, ATM, PRSS1 genes and the hereditary colon cancer (Lynch syndrome) genes, are associated with significantly increased risk of PC. ${ }^{19}$ Mutations in these genes explain only $\sim 10 \%$ of the familial susceptibility to PC. Individuals with PC susceptibility gene mutations may not have many affected family members. Patients with apparently sporadic pancreatic cancer can have mutations in BRCA2, as can those without a family history of breast, ovarian cancer. ${ }^{20}$ Incomplete or low penetrance is a common feature of familial PC susceptibility gene mutations.

Patients with Peutz-Jeghers syndrome, regardless of family history, should be considered for screening (agree 96\%, grade moderate, 'do it').

Patients with Peutz-Jeghers syndrome (who generally carry germline STK11 gene mutations) have a very high (132-fold ${ }^{21}$ ) 
risk of PC. Lifetime cumulative risk to age 65-70 for PC in patients with Peutz-Jeghers syndrome is $11-36 \%{ }^{22}$

BRCA2 mutation carriers with one or more affected FDR with PC (agree $85.7 \%$, grade low, 'probably do it') and those with two or more affected family members (even without a FDR) (agree $89.8 \%$, grade low, 'probably do it') should be considered for screening.

Germline BRCA2 gene mutations account for the highest percentage of known causes of inherited PC. These have been identified in $5-17 \%$ of familial PC kindreds. ${ }^{23-25}$ The RR of PC in BRCA2 gene mutation carriers is $3.5(95 \%$ CI 1.87 to 6.58). ${ }^{26} 27$ Individuals with Jewish ancestry and a family history of PC should be considered for genetic counselling and testing for the founder BRCA2 gene mutation, 6174delT, present in $1 \%$ of Ashkenazi Jewish individuals ${ }^{28}$ and $4 \%$ of patients with PC. ${ }^{29}$ It has not been established that the risk of $\mathrm{PC}$ in BRCA1 gene mutation carriers is increased. One cohort study found a modest increased risk of pancreatic cancer $(R R=2.3) .{ }^{30}$ No agreement was reached on the question of screening $B R C A 2$ mutation carriers with no family history of PC (agree $51.1 \%$ ), or for BRCA1 mutation carriers with one affected FDR or two affective relatives but no FDR (agree $69.4 \%)$

PALB2 mutation carriers with one or more affected FDR with PC should be screened (agree $77.5 \%$, grade very low, "probably do it').

PALB2 (partner and localiser of BRCA2) was recently identified as a PC susceptibility gene. ${ }^{31}$ Germline mutations have been detected in up to $3 \%$ of patients with familial PC. ${ }^{31-35}$ The magnitude of PC risk in PALB2 mutation carriers has not been established. However, given the function of the PALB2 gene, the risk of PC among PALB2 gene mutation carriers is estimated to be similar to that found for $B R C A 2$ gene mutation carriers.

p16 mutation carriers with one or more affected FDR with PC should be considered for screening. (agree $87.8 \%$, grade low, (probably do it').

Germline p 16 gene mutations are found in families with familial atypical multiple mole melanoma syndrome (FAMMM syndrome), an autosomal dominant disease with variable penetrance. PC risk among p 16 gene mutation carriers is estimated to be increased 13- to 22 -fold, compared with the general population. $^{36-38}$

Patients with Lynch syndrome and one affected FDR with PC should be considered for screening. (agree $87.5 \%$, grade low, (probably do it').

Patients with mismatch repair gene (MLH1, MSH2, MSH6, PMS2) gene mutations (Lynch syndrome) have an estimated lifetime risk of $3.7 \%$ of developing PC (8.6-fold higher risk). ${ }^{39} 40$ Patients with PC having histology characteristic of mismatch repair-deficient cancers ('medullary' histology) ${ }^{41}$ should have their pedigree evaluated for possible hereditary non-polyposis colorectal cancer.

The estimated lifetime risk of PC in individuals with hereditary pancreatitis is high (about $40 \%$ ). ${ }^{42}$ Many of these individuals have germline PRSS1 gene mutations. This PC risk is directly related to the duration of recurrent pancreatitis and chronic inflammation. ${ }^{43}$ Screening of PRSS1 mutation carriers with longstanding chronic pancreatitis is being performed within established programmes ${ }^{44}$ but it is controversial whether healthy siblings with a PRSS1 mutation should also be screened.

\section{At what age should screening begin and end?}

There was disagreement about the age to initiate screening in HRIs (Appendix). For individuals with familial PC, the average age at diagnosis is $68 .{ }^{18}$ Fifty-one per cent voted to recommend starting screening at age 50 . In contrast, screening typically begins at age 40 in PRSS1 mutation carriers with hereditary pancreatitis owing to younger age of onset of PC. ${ }^{45}$ Smokers with a family history of PC have a greater risk of developing PC than non-smokers, ${ }^{18}$ but there was no consensus as to whether to recommend initiating screening at an earlier age for current smokers (Appendix). There was also no consensus recommendation about the age to end screening for HRIs without pancreatic lesions (Appendix).

\section{How should high-risk individuals be screened?}

Published screening studies have employed different screening tests. Direct interpretation of screening modalities is limited by differences in study populations, and reported diagnostic yields have ranged between $1.3 \%$ and $50 \%$, depending on whether resected neoplasms or pancreatic lesions were tabulated (table 1). ${ }^{246-9344647}$ Of 1040 HRIs screened, to date only 70 $(6.7 \%)$ had a pancreatic lesion or suspected neoplasm resected. $^{24}$ 6-9 344647 Small cysts (branch-duct intraductal papillary mucinous neoplasms or BD-IPMNs) are the most common abnormality detected (in 34\% and 53\% of HRIs aged 50-59 and 60-69, respectively ${ }^{11}$ ). Solid masses are rarely detected (20 of 70 resected were pancreatic ductal adenocarcinomas. $^{2} 46-9344647$ The number of incident PCs detected in published studies is likely to be unreported (only four were cohort studies, all with limited follow-up), but eight of 20 $(40 \%)$ of the PCs diagnosed in screened HRIs were not detected at baseline screening.

Initial screening should include (multiple answers allowed) endoscopic ultrasonography (EUS) (agree $83.7 \%$, grade moderate, 'do it'), MRI/magnetic resonance cholangiopancreatography (MRCP) (agree $73.5 \%$, grade moderate, 'do it'), CT (26.5\%), abdominal ultrasound (14.3\%), endoscopic retrograde cholangiopancreatography (ERCP) (2\%).

EUS and MRI are considered the most accurate tools for pancreatic imaging and do not involve ionising radiation. Few studies have compared the diagnostic yield of imaging tests for HRIs in screening, and most comparisons have not been performed in a blinded, randomised fashion. The prospective CAPS3 study (published after the CAPS summit) performed blinded comparisons of standardised pancreatic-protocol CT, secretin-enhanced MRI/MRCP and EUS for one-time screening. ${ }^{11}$ It showed that EUS and MRI are better than CT for the detection of small, predominantly cystic, pancreatic lesions, with good to excellent concordance of lesion number, size and location between EUS and MRI/MRCP. EUS, MRI/MRCP and CT identified pancreatic lesions in $42.6 \%, 33.3 \%$ and $11 \%$ of screened HRIs, respectively. ${ }^{11}$ MRCP provided the best visualisation of cyst communication with the main pancreatic duct.

Incorrect diagnosis of lesions identified by EUS and/or MRI is a significant concern, particularly in the screening process. Some cysts are found at resection to be benign serous cystadenomas, while other resected pancreata have only low-grade pancreatic intraepithelial neoplasia (PanIN) associated with lobulocentric parenchymal atrophy. ${ }^{4-7} 34$ These results highlight the risk of overtreatment using available screening tests. 
The risk of overtreatment for pancreatic screening is magnified by the risks of morbidity and mortality ( $1-2 \%)$ of pancreatic surgery. The risk of incorrect diagnosis is particularly true for EUS, an operator-dependent test with only modest interobserver agreement. ${ }^{48}$

There was excellent agreement that radiation exposure and the suboptimal detection rate preclude CT from being a routine pancreatic screening test. ${ }^{2} 1146$ Abdominal ultrasound and ERCP were also not recommended for screening, owing to their low diagnostic sensitivity and the risk of pancreatitis, respectively.

\section{Additional tests}

ERCP should be performed as an additional test if a solid lesion (disagree $77.5 \%$, grade high, 'don't do it') or cystic lesion (disagree $77.5 \%$, grade moderate, 'don't do it') is detected. When a solid lesion is detected, CT should be performed (agree 75.5\%, grade, low, 'do it').

When ERCP was performed routinely for abnormal EUS results, it did not improve diagnostic yield and was associated with a $7 \%$ pancreatitis rate. ${ }^{4}$

No consensus was reached on the role of EUS-guided fineneedle aspiration (FNA) to evaluate solid or cystic lesions in asymptomatic HRIs (Appendix). The role of EUS-FNA in the clinical management of most pancreatic cysts is limited, given the low accuracy of cytology in cystic lesions, ${ }^{49} 50$ and the low volume of cyst fluid aspirated from small cysts. False-positive cytology from subcentimetre solid indeterminate lesions may also lead to unnecessary surgery. ${ }^{59}$

Multidetector pancreatic-protocol CT was recommended for evaluation of solid lesions identified by EUS or MRI. The level of evidence that supports this agreement is low.

\section{Surveillance}

For routine follow-up, the best imaging test is (multiple answers allowed): EUS (79.6\%), MRI/MRCP (69.4\%), CT (22.4\%), abdominal ultrasound (4.1\%), ERCP (2\%).

How should surveillance be performed after baseline screening? Published studies have generally used the same imaging tests for follow-up as for baseline imaging. There was no consensus reached on the ideal screening interval in the absence of pancreatic abnormalities at baseline, but $73.5 \%$ of participants suggested a 12-month interval. There is only indirect and limited evidence to support this recommendation. The vast majority of individuals in whom a clinically relevant lesion developed during follow-up had pancreatic abnormalities at baseline. ${ }^{4} 9$ Furthermore, HRIs who presented with an advanced pancreatic malignancy after prior normal or indeterminate imaging were diagnosed $\geq 12$ months later. ${ }^{2} 9$

\footnotetext{
Patients with a non-suspicious cyst should have an imaging test after 6-12 months (agree 83.7\%, grade moderate, 'do it'). Patients with a newly detected indeterminate solid lesion should have follow-up screening at 3 months, if surgery is not imminent (agree $85.7 \%$, grade low, 'do it'). If an indeterminate main pancreatic duct stricture is detected, repeat imaging should be performed within 3 months (agree 95.9\%, grade low, 'do it').
}

Cystic branch-duct lesions (presumed BD-IPMNs) without concerning features indicating malignancy ${ }^{51}$ should be re-evaluated at intervals depending on size, similar to accepted international consensus guidelines for sporadic BD-IPMNs. ${ }^{51}$ The majority of such BD-IPMNs remained stable during follow-up. 267934
Small ( $<1 \mathrm{~cm}$ diameter) lesions identified as solid by EUS are difficult to manage, particularly when not detected by MRI or CT. These lesions can be aspirated but the yield for these lesions is low. Some indeterminate solid lesions identified only by EUS are cancers, but they can be benign lesions, such as non-metastatic pancreatic neuroendocrine tumours ${ }^{2} 411$ or lowgrade PanIN with focal associated lobulocentric parenchymal atrophy. ${ }^{5}$ There was no consensus reached on the need for additional tests such as CT, ERCP, FNA, or timing of repeat imaging (although $73.5 \%$ suggested 3 months) to evaluate these lesions (Appendix).

Long-term follow-up of PC screening cohorts is lacking, (maximum follow-up period; 10 years, ${ }^{9}{ }^{34}$ mean follow-up time of $4^{9}$ to 4.2 years $^{2}$ ). Importantly, the group acknowledged that until there are additional studies we will not know if screening HRIs saves lives.

\section{When should surgery be performed? What type of surgery should be performed?}

Screening should only be offered to individuals who are candidates for surgery (agree $75.5 \%$, grade moderate, 'do it'). Pancreatic resections should be performed at high-volume specialty centres (agree 100\%, grade moderate, 'do it'). Determining when surgery is required for pancreatic lesions is difficult and is best individualised after multidisciplinary assessment, preferably within research studies.

There is little consensus about which lesions detected by screening require surgery. The few published reports are based on limited numbers of patients. ${ }^{52}{ }^{53}$ Because of the risks of pancreatectomy, prophylactic surgery is not recommended for asymptomatic HRIs without an identifiable lesion. When indicated, pancreatic surgery is best performed at a high-volume specialty centre. Multiple studies have shown volume directly correlates with outcomes. ${ }^{54} 55$

Unambiguous solid lesions $(\geq 1 \mathrm{~cm}$, or seen by multiple imaging modalities) are ominous and the threshold for removing them is much lower. There was no consensus as to whether any indeterminate solid lesions detected by EUS should be resected (Appendix).

The majority of cystic lesions detected by screening appear to be low-risk branch-duct IPMNs (table 1). The Sendai international consensus guidelines have been developed and updated $^{56}$ to help stratify patients with an IPMN as low risk versus high risk for either developing or currently harbouring a malignancy. ${ }^{51}$ In subjects with suspected BD-IPMNs, resection is considered if the patient has symptoms attributable to the cyst(s), if the cysts are $>3 \mathrm{~cm}$ in size, or if the cysts contain mural nodules. Logic would dictate that if these are the recommendations for subjects without a strong family history of PC, then these thresholds for resection should be either the same or lower in subjects with a strong family history. There was no consensus on the size criterion for resection of suspected BD-IPMNs or other cysts in HRIs but the majority agreed that surgery should be considered for suspected BD-IPMNs which were $\geq 2 \mathrm{~cm}$ (Appendix). Pathologically confirmed PanIN-3 lesions have been found in the pancreata of individuals who had resections of IPMNs smaller than $1 \mathrm{~cm} .{ }^{5}{ }^{11}$ High-grade dysplasia and main-duct involvement have been identified at resection of some individuals who had surgery for one or more small $(<3 \mathrm{~cm})$ BD-IPMNs. ${ }^{4} 51134$ However, there is insufficient evidence to lower the threshold criteria for surgery for patients with lesions identified during pancreatic screening. 
Management of patients with resected lesions was discussed, particularly how the preliminary and final pathology results, including margin status, should influence operative treatment.

Intraoperatively, further pancreatectomy (including total pancreatectomy) should generally be performed to achieve R0 resection of cancer (agree $75.5 \%$, grade low, 'do it'). Intraoperatively, further pancreatectomy (including total pancreatectomy) should not be performed on patients with only unifocal PanIN-2 in the resected specimen, (agree $77.6 \%$, grade low, 'don't do it'). The presence of PanIN-3 at the margin should be dealt with in consideration of the overall medical condition and life expectancy of the patient. The presence of PanIN-2, low-grade IPMN or intermediate-grade IPMN (on either frozen or permanent sections) at the margin or in the resection specimen should not drive further resection.

In patients undergoing surgery for invasive PC, complete resection of the cancer is recommended. If only PanIN is at the margin, it is considered unlikely that resection of additional parenchyma would be beneficial, even if the PanIN is high grade. ${ }^{57}$ Importantly, it is difficult to grade PanIN in intraoperative frozen sections.

Postoperatively, further resection of the pancreas to remove PanIN-2 at the margin should be performed in high-risk patients without PC (disagree 79.5\%, grade low, 'don't do it'). Postoperatively, further resection of the pancreas should be performed because unifocal PanIN-2 (disagree $81.6 \%$, grade low, 'don't do it') or multifocal PanIN-2 (disagree $77.5 \%$, grade low, 'don't do it') was found anywhere in the resected specimen.

Multiple scenarios for consideration of further pancreatectomy after R0 resection of cancer did not reach consensus agreement, including management of PanIN-3 at the margin (Appendix). PanIN-3 at the resection margin in non-familial patients treated for PC does not significantly affect the postoperative course. ${ }^{57}$ However, follow-up imaging was recommended less than 6 months after surgery if there was any PanIN-3 in the resected pancreas of individuals without PC.

\section{What are the goals of screening? What outcome(s) would be considered a 'success'?}

Screening HRIs can be considered successful if it can be shown that the benefits outweigh the costs of screening. The goal of screening is the reduction of pancreatic cancer-related mortality. Evidence for success is best provided by large randomised controlled trials in which the outcomes of subjects who undergo surveillance are compared with appropriate controls, as has been demonstrated for colonoscopy screening. ${ }^{58}$ However, given the relatively low incidence of familial PC, such trials are difficult to undertake. Surrogate end points that define the success of pancreatic screening are therefore needed. Pathological staging is critical and a standard protocol for handling pancreatic resection specimens is recommended (Appendix). The following questions (What are the goals of screening? What outcome(s) would be considered a success?) were designed to define surrogate end points of screening, such as resection of potentially curable lesions (high-grade precursor neoplasms and early invasive carcinomas), as these lesions, if left untreated, can progress to incurable and lethal disease.

One target for early detection and treatment is resectable carcinoma (agree $83.7 \%$, grade high, 'do it'). Detection and treatment of early invasive cancer (T1N0M0) (agree $89.8 \%$, grade high, 'do it') at baseline or follow-up (agree 77.5\%, grade moderate, 'do it') should be considered a success. Detection and treatment of any invasive resectable PC at baseline screening should also be considered a success of the screening programme (agree $89.8 \%$, grade high, 'do it').

Long-term survival can be achieved by resecting small nonmetastatic PCs, ${ }^{59} 60$ particularly if margins are negative for invasive PC (R0 resection). ${ }^{57}{ }^{61}$ However, most patients who undergo an $\mathrm{R} 0$ resection of their pancreatic cancer will die from their disease. Survival is most likely for patients with the smallest cancers (T1N0M0). A critical statistic which screening and surveillance programmes should track is the number of highrisk patients that need to be screened and treated, ${ }^{62}$ which considers the likelihood that treatment will prevent the target event of PC at the expense of adverse events. In one prospective screening study of high-risk Leiden $p 16$ mutation carriers using $\mathrm{MRI} / \mathrm{MRCP}$, nine invasive pancreatic PCs were detected and treated in 79 patients followed up for a median of 4 years. The number of patients needed to be screened to detect and treat one PC was 11.9

Well-differentiated neuroendocrine tumours (PanNETs) have been detected within familial PC screening programmes. ${ }^{2} 411$ PanNETs $<0.5 \mathrm{~cm}$ (microadenomas) are essentially benign lesions. Resection of PanNETs between 0.5 and $1.0 \mathrm{~cm}$ is generally curative. ${ }^{63}$ There was no consensus as to whether detecting and treating PanNETs should be considered a success of screening (Appendix).

One potential target for early detection is PanIN (agree $81.7 \%$, grade high, 'do it'). Detecting and treating multifocal PanIN-3 should be considered a success (agree $83.7 \%$, grade moderate, 'do it'). Whether to detect and treat unifocal PanIN-3 did not reach consensus (agree $73.5 \%$ )

The strength of the evidence linking sporadic PanIN-3 lesions to invasive carcinoma is based on clinical associations and genetic analyses. ${ }^{64-66}$ Similarly, strong evidence supports the hypothesis that some of the invasive PCs that arise in patients with a family history of pancreatic cancer arise from PanIN lesions. ${ }^{67} 68$ Although PanINs are a well-accepted precursor of sporadic and familial PC, the frequency and rate at which PanINs progress to invasive carcinoma is not known. In a nonfamilial population, it is estimated that the average adult pancreas has five PanINs and that $0.86 \%$ of these progress to invasive cancer. $^{69}$ It may take a decade or more for an early precursor cell (a low-grade PanIN) to progress to PC, and initial estimates suggest that the first invasive cancer cell may take several years to extend beyond the pancreas or metastasize. Importantly, the 'window' for clinical detection of an invasive PC is shorter since these lesions are only detected once they reach a certain size. ${ }^{70}$ These estimates may not apply to patients with a strong family history of PC, especially those with specific genetic mutations that may increase the rate at which precursor lesions progress.

Another target for early detection and treatment is IPMN (agree $87.7 \%$, evidence high, 'do it'). Detection and treatment of IPMN with high-grade dysplasia should be considered a success of a screening/surveillance programme (agree 95.9\%, evidence high, 'do it').

IPMNs, particularly IPMNs with high-grade dysplasia, are associated with a significantly increased risk of invasive PC. 67687172 The IPMN phenotype has been described in familial PC relatives and gene mutation carriers. ${ }^{73}$ The frequency and rate at which IPMNs in HRIs progress to invasive PC are not well known. In patients with apparently sporadic noninvasive IPMNs it may take 3-5 years for a clinically detectable non-invasive lesion to progress to an invasive $\mathrm{PC}^{74}$ 
Furthermore, in patients with small BD-IPMN(s) followed up over 5 years, only $2.4-6.9 \%$ of these lesions progressed to invasive PC. 7576

\section{Important areas where there was lack of consensus: areas for future research}

Topics that did not reach consensus voting are listed in the Appendix. Additional evidence is required to more accurately answer important questions, such as who to screen, when to begin screening and the frequency of screening. Some of the important gaps in knowledge pertain to the optimal age at which to begin screening, the role of as yet unidentified PC susceptibility genes as a guide to optimising screening and how to incorporate environmental risk factors such as smoking, diabetes, obesity and other exposures into risk stratification.

The CAPS summit recommended prioritising research into areas where there was lack of consensus: diagnostic evaluation and management of cystic and solid lesions detected by screening and postoperative management. Importantly, the group recommends collaborative multicentre institutional review board-approved studies to collect data on demographics, family history, risk factors, and to bank tissue, juice, aspirated fluid and blood to improve biomarker prediction of the risk of progression to $\mathrm{PC}$ in HRIs. Ultimately, research into how to improve screening methods, the outcomes of screening and surveillance for PC and the cost-effectiveness of alternative approaches, is of the highest priority.

\section{SUMMARY}

Screening studies have identified pancreatic neoplasms in asymptomatic patients with strong family histories of PC. However, available evidence supporting screening and surveillance is limited to observational studies. The diagnostic yield from pancreatic screening depends on many factors, including the extent of an individual's family history, the age at which screening begins and the screening modality used. Screening may also lead to the discovery of incidental or indeterminate lesions, resulting in diagnostic confusion and uncertain management. There is a clear need to improve approaches to screening of HRIs. The management of asymptomatic pancreatic lesions detected by imaging tests remains the most challenging aspect of screening and surveillance programmes. Individualised decision-making within multidisciplinary programmes and prospective research studies is essential. The findings of this workgroup should standardise current efforts and serve as a platform for the development of future multidisciplinary research protocols.

\footnotetext{
Author affiliations

${ }^{1}$ Department of Medicine (Gastroenterology), Johns Hopkins Medical Institutions, Baltimore, Maryland, USA

${ }^{2}$ Department of Gastroenterology, Erasmus MC, University Medical Center, Rotterdam, The Netherlands

${ }^{3}$ Department of Pathology, Johns Hopkins Medical Institutions, Baltimore, Maryland, USA

${ }^{4}$ Department of Pathology, University Medical Center, Utrecht, The Netherlands ${ }^{5}$ Department of Radiology, Johns Hopkins Medical Institutions, Baltimore, Maryland, USA

${ }^{6}$ Department of Radiology, Academic Medical Center, Amsterdam, The Netherlands ${ }^{7}$ Department of Surgery, Johns Hopkins Medical Institutions, Baltimore, Maryland, USA

${ }^{8}$ Department of Surgery, University of Verona, Verona, Italy

${ }^{9}$ Department of Oncology, Academic Medical Center, Amsterdam, The Netherlands

${ }^{10}$ Division of Gastroenterology, Mayo Clinic, Rochester, Minnesota, USA

${ }^{11}$ Division of Gastroenterology, University Hospitals of Cleveland, Cleveland Ohio, USA

${ }^{12}$ Department of Gastroenterology, Academic Medical Center, Amsterdam,

The Netherlands
}

Acknowledgements We thank Sandy Markowitz for her assistance in the organisation and coordination of the CAPS Summit.

Collaborators CAPS International Consortium Summit participants: Paolo Giorgio Arcidiacono (Milan, Italy), Detlef Bartsch (Marburg, Germany), Katharina Biermann (Rotterdam, The Netherlands), Terri Brentnall (Washington, USA), Amitabh Chak (Ohio, USA), Petr Dite (Brno, Czech Republic), Timothy Donahue (California, USA), Dayna Early (Missouri, USA), James Farrell (California, USA), Carlos Fernandez-Del Castillo (Massachusetts, USA), Harold Frucht (New York, USA), Noriyoshi Fukushima (Tochigi, Japan), Jenny Geurts (Wisconsin, USA), Pascal Hamell (Clichy, France), Julio Iglesias-Garcia (Santiago de Compostela, Spain), Alison Klein (Maryland, USA), Guenter Kloeppel (Munich, Germany), Jesse Lachter (Haifa, Israel), Peter Langer (Marburg, Germany), Jeffrey Lee (Texas, USA), Michael Levy (Minnesota, USA), Hiroyuki Maguchi (Sapporo, Japan), Daniel Margolis (Los Angeles, USA), Takao Ohtsuka (Fukuoka, Japan), Sara Olson (New York, NY), Gloria Petersen (Minnesota, USA), Thomas Savides (California, USA), Sapna Syngal (Massachusetts, USA), Eric Tamm (Texas, USA), Masao Tanaka (Fukuoka, Japan), Hans Vasen (Leiden, The Netherlands), Anja Wagner (Erasmus, The Netherlands), Huamin Wang (Texas, USA), David Williams (Sydney, Australia), Kenjii Yamao (Nagoya, Japan).

Contributors MIC - was the project principal investigator, helped obtain funding and supervised the overall conduct of the conference; developed the conference concept and programme, served as gastroenterology workgroup leader, assisted with the acquisition, analysis and interpretation of data, drafting and critical revision of the manuscript. FH—constructed the online survey, assisted with the acquisition of data, analysis and interpretation of data and drafting and critical revision of the manuscript. $\mathrm{RHH}$ - assisted with obtaining grant support, assisted with the conference concept and programme, served as pathology workgroup leader and helped with the drafting and critical revision of the manuscript. GJO — served as pathology workgroup leader and participated in the drafting of the manuscript. J-WP-assisted with the development of the conference concept and programme, served as gastroenterology workgroup leader and helped with the drafting and critical revision of the manuscript. $\mathrm{PF}$ - served as gastroenterology workgroup leader and helped with the drafting of the manuscript. IK-assisted with the conference concept and design, assisted with presentation of scientific evidence, served as radiology workgroup leader and helped with the drafting of the manuscript. YN- served as radiology workgroup leader and helped with the drafting of the manuscript. RSS - assisted with the conference concept and design, assisted with presentation of scientific evidence, served as surgery workgroup leader and helped with the drafting and critical revision of the manuscript. CB - served as surgery workgroup leader and helped with the drafting and revision of the manuscript. IKI—assisted with the study concept and design, assisted with acquisition of data, served as genetics/epidemiology workgroup leader and helped with the drafting and revision of the manuscript. MJL-helped with the drafting and critical revision of the manuscript. $\mathrm{AC}$ - helped with drafting and critical revision of the manuscript. MG - assisted with obtaining grant and material support, assisted with the study concept and design, served as genetics/epidemiology workgroup leader, assisted with acquisition of data and helped with the drafting and critical revision of the manuscript. MB - served as co-director of the conference, helped obtain funding and supervised the overall conduct of the conference; helped develop the conference concept and programme, assisted with the interpretation of data and helped with the drafting and critical revision of the manuscript.

Funding European Association for Gastroenterology, Endoscopy and Nutrition (EAGEN), Olympus Corporation, Myriad Corporation, John and Peter Hooven Endowment and The Sol Goldman Pancreatic Cancer Research Center. Role of funding sources: All sponsors had no role in planning the content or conduct of the conference, analysis of the results and preparation of the manuscript.

Competing interests $\mathrm{RHH}$ and MG receive royalty payments from Myriad Genetics. $\mathrm{MB}$ is council member and treasurer of EAGEN. All other coauthors have no relevant competing interests or financial disclosures.

Provenance and peer review Not commissioned; externally peer reviewed.

Open Access This is an Open Access article distributed in accordance with the Creative Commons Attribution Non Commercial (CC BY-NC 3.0) license, which permits others to distribute, remix, adapt, build upon this work non-commercially, and license their derivative works on different terms, provided the original work is properly cited and the use is non-commercial. See: http://creativecommons.org/licenses/by-nc/3.0/

\section{REFERENCES}

1. Jemal A, Bray F, Center MM, et al. Global cancer statistics. CA Cancer J Clin 2011:61:69-90.

2. Al-Sukhni W, Borgida A, Rothenmund $\mathrm{H}$, et al. Screening for Pancreatic Cancer in a High-Risk Cohort: An Eight-Year Experience. J Gastrointest Surg 2011;16:771-83.

3. Brentnall TA. Pancreatic cancer surveillance: learning as we go. Am J Gastroenterol 2011;106:955-6.

4. Canto MI, Goggins M, Hruban RH, et al. Screening for early pancreatic neoplasia in high-risk individuals: a prospective controlled study. Clin Gastroenterol Hepatol 2006;4:766-81; quiz 665. 
5. Canto M, Goggins $\mathbf{M}$, Yeo $\mathbf{C}$, et al. Screening for pancreatic neoplasia in high-risk individuals. Clin Gastro Hepatol 2004;2:606-21.

6. Langer $\mathbf{P}$, Kann $\mathrm{PH}$, Fendrich $\mathrm{V}$, et al. Five years of prospective screening of high-risk individuals from families with familial pancreatic cancer. Gut 2009;58:1410-18.

7. Ludwig E, Olson SH, Bayuga $\mathrm{S}$, et al. Feasibility and yield of screening in relatives from familial pancreatic cancer families. Am J Gastroenterol 2011:106:946-54.

8. Poley JW, Kluijt I, Gouma DJ, et al. The yield of first-time endoscopic ultrasonography in screening individuals at a high risk of developing pancreatic cancer. Am J Gastroenterol 2009:104:2175-81.

9. Vasen HF, Wasser M, van Mil A, et al. Magnetic Resonance Imaging Surveillance Detects Early-Stage Pancreatic Cancer in Carriers of a p16-Leiden Mutation. Gastroenterology 2011;140:850-6.

10. Verna EC, Hwang C, Stevens PD, et al. Pancreatic Cancer Screening in a Prospective Cohort of High-Risk Patients: A Comprehensive Strategy of Imaging and Genetics. Clin Cancer Res 2010;16:5028-37.

11. Canto MI, Hruban RH, Fishman EK, et al. Frequent Detection of Pancreatic Lesions in Asymptomatic High-Risk Individuals. Gastroenterology 2012.

12. Atkins D, Best D, Briss PA, et al. Grading quality of evidence and strength of recommendations. BMJ 2004;328:1490-4.

13. Guyatt GH, Oxman AD, Vist GE, et al. GRADE: an emerging consensus on rating quality of evidence and strength of recommendations. BMJ 2008;336:924-6.

14. Jaeschke R, Guyatt GH, Dellinger P, et al. Use of GRADE grid to reach decisions on clinical practice guidelines when consensus is elusive. BMJ 2008;337:a744.

15. Wang W, Chen S, Brune KA, et al. PancPRO: risk assessment for individuals with a family history of pancreatic cancer. J Clin Oncol 2007;25:1417-22.

16. Klein AP, Brune KA, Petersen GM, et al. Prospective risk of pancreatic cancer in familial pancreatic cancer kindreds. Cancer Res 2004;64:2634-8.

17. Grover S, Syngal S. Hereditary Pancreatic Cancer. Gastroenterology 2010:139:1076-80

18. Brune KA, Lau B, Palmisano $E$, et al. Importance of age of onset in pancreatic cancer kindreds. J Natl Cancer Inst 2010:102:119-26.

19. Hruban $\mathbf{R H}$, Canto Ml, Goggins $\mathrm{M}$, et al. Update on familial pancreatic cancer. Adv Surg 2010;44:293-311

20. Goggins M, Schutte M, Lu J, et al. Germline BRCA2 gene mutations in patients with apparently sporadic pancreatic carcinomas. Cancer Res 1996;56:5360-4.

21. Giardiello FM, Brensinger JD, Tersmette AC, et al. Very high risk of cancer in familial Peutz-Jeghers syndrome. Gastroenterology 2000:119:1447-53.

22. van Lier MG, Wagner A, Mathus-Vliegen EM, et al. High cancer risk in Peutz-Jeghers syndrome: a systematic review and surveillance recommendations. Am J Gastroentero/ 2010;105:1258-64; author reply 65.

23. Couch FJ, Johnson MR, Rabe KG, et al. The prevalence of BRCA2 mutations in familial pancreatic cancer. Cancer Epidemiol Biomarkers Prev 2007:16:342-6.

24. Hahn SA, Greenhalf B, Ellis I, et al. BRCA2 germline mutations in familial pancreatic carcinoma. J Natl Cancer Inst 2003:95:214-21.

25. Murphy KM, Brune KA, Griffin C, et al. Evaluation of candidate genes MAP2K4, MADH4, ACVR1B and BRCA2 in familial pancreatic cancer: deleterious BRCA2 mutations in 17\%. Cancer Res 2002;62:3789-93.

26. Yilmaz EN, van Heek NT, van der Spoel Jl, et al. Myocardial contusion as a result of isolated sternal fractures: a fact or a myth? Eur J Emerg Med 1999;6:293-5.

27. van Asperen CJ, Brohet RM, Meijers-Heijboer EJ, et al. Cancer risks in BRCA2 families: estimates for sites other than breast and ovary. J Med Genet 2005:42:711-19.

28. Struewing JP, Abeliovich D, Peretz T, et al. The carrier frequency of the BRCA 185delAG mutation is approximately 1 percent in Ashkenazi Jewish individuals. Nat Genet 1995;11:198-200.

29. Ferrone CR, Levine DA, Tang $\mathrm{LH}$, et al. BRCA germline mutations in Jewish patients with pancreatic adenocarcinoma. J Clin Oncol 2009;27:433-8.

30. Thompson D, Easton DF. Cancer Incidence in BRCA1 mutation carriers. J Nat Cancer Inst 2002;94:1358-65.

31. Jones $\mathbf{S}$, Hruban RH, Kamiyama $\mathrm{M}$, et al. Exomic sequencing identifies PALB2 as a pancreatic cancer susceptibility gene. Science 2009;324:217.

32. Slater EP, Langer P, Niemczyk E, et al. PALB2 mutations in European familial pancreatic cancer families. Clin Genet 2010;78:490-4.

33. Tischkowitz MD, Sabbaghian N, Hamel N, et al. Analysis of the gene coding for the BRCA2-interacting protein PALB2 in familial and sporadic pancreatic cancer. Gastroenterology 2009;137:1183-6.

34. Schneider R, Slater EP, Sina M, et al. German national case collection for familial pancreatic cancer (FaPaCa): ten years experience. Fam Cancer 2011;10:323-30.

35. Harinck F, Kluijt I, van Mil SE, et al. Routine testing for PALB2 mutations in familia pancreatic cancer families and breast cancer families with pancreatic cancer is not indicated. Eur J Hum Genet 2012:20:577-9.

36. Goldstein AM, Chan $M$, Harland $M$, et al. Features associated with germline CDKN2A mutations: a GenoMEL study of melanoma-prone families from three continents. J Med Genet 2007;44:99-106.

37. Lynch HT, Fusaro RM, Lynch JF, et al. Pancreatic cancer and the FAMMM syndrome. Fam Cancer 2008;7:103-12.
38. de Snoo FA, Bishop DT, Bergman W, et al. Increased risk of cancer other than melanoma in CDKN2A founder mutation (p16-Leiden)-positive melanoma families. Clin Cancer Res 2008:14:7151-7.

39. Win AK, Young JP, Lindor NM, et al. Colorectal and other cancer risks for carriers and noncarriers from families with a DNA mismatch repair gene mutation: a prospective cohort study. J Clin Oncol 2012;30:958-64.

40. Kastrinos F, Mukherjee $\mathrm{B}$, Tayob N, et al. Risk of pancreatic cancer in families with Lynch syndrome. JAMA 2009;302:1790-5.

41. Goggins $\mathbf{M}$, Offerhaus GJ, Hilgers $\mathbf{W}$, et al. Pancreatic adenocarcinomas with DNA replication errors $(\mathrm{RER}+)$ are associated with wild-type K-ras and characteristic histopathology. Poor differentiation, a syncytial growth pattern and pushing borders suggest RER +. Am J Pathol 1998;152:1501-7.

42. Lowenfels AB, Maisonneuve P, DiMagno EP, et al. Hereditary pancreatitis and the risk of pancreatic cancer. International Hereditary Pancreatitis Study Group. J Natl Cancer Inst 1997;89:442-6.

43. Whitcomb DC. Inflammation and Cancer V. Chronic pancreatitis and pancreatic cancer. Am J Physiol Gastrointest Liver Physiol 2004;287:G315-19.

44. Vitone LJ, Greenhalf W, Howes NR, et al. Hereditary pancreatitis and secondary screening for early pancreatic cancer. Rocz Akad Med Bialymst 2005; 50:73-84.

45. Ulrich CD. Pancreatic cancer in hereditary pancreatitis: consensus guidelines for prevention, screening and treatment. Pancreatology 2001;1:416-22.

46. Brentnall TA, Bronner MP, Byrd DR, et al. Early diagnosis and treatment of pancreatic dysplasia in patients with a family history of pancreatic cancer. Ann Intern Med 1999:131:247-55.

47. Kimmey MB, Bronner MP, Byrd DR, et al. Screening and surveillance for hereditary pancreatic cancer. Gastrointest Endosc 2002:56:S82-6.

48. Topazian M, Enders F, Kimmey M, et al. Interobserver agreement for EUS findings in familial pancreatic-cancer kindreds. Gastrointest Endosc 2007;66:62-7.

49. Brugge WR, Lewandrowski K, Lee-Lewandrowski E, et al. Diagnosis of pancreatic cystic neoplasms: a report of the cooperative pancreatic cyst study. Gastroenterology 2004;126:1330-6.

50. Cizginer S, Turner B, Bilge AR, et al. Cyst fluid carcinoembryonic antigen is an accurate diagnostic marker of pancreatic mucinous cysts. Pancreas 2011:40:1024-8.

51. Tanaka M, Chari S, Adsay V, et al. International consensus guidelines for management of intraductal papillary mucinous neoplasms and mucinous cystic neoplasms of the pancreas. Pancreatology 2006;6:17-32.

52. Del Chiaro M, Zerbi A, Capurso G, et al. Familial pancreatic cancer in Italy. Risk assessment, screening programs and clinical approach: a position paper from the Italian Registry. Dig Liver Dis 2010;42:597-605.

53. Jacobs EJ, Chanock SJ, Fuchs CS, et al. Family history of cancer and risk of pancreatic cancer: a pooled analysis from the Pancreatic Cancer Cohort Consortium (PanScan). Int J Cancer 2010;127:1421-8.

54. Finks JF, Osborne NH, Birkmeyer JD. Trends in hospital volume and operative mortality for high-risk surgery. N Engl J Med 2011;364:2128-37.

55. Lemmens VE, Bosscha K, van der Schelling G, et al. Improving outcome for patients with pancreatic cancer through centralization. Br J Surg 2011;98:1455-62

56. Tanaka M, Fernandez-Del Castillo C, Adsay V, et al. International consensus guidelines 2012 for the management of IPMN and MCN of the pancreas. Pancreatology 2012;12:183-97

57. Matthaei H, Hong SM, Mayo SC, et al. Presence of pancreatic intraepithelia neoplasia in the pancreatic transection margin does not influence outcome in patients with R0 resected pancreatic cancer. Ann Surg Oncol 2011:18:3493-9.

58. Zauber AG, Winawer SJ, O'Brien MJ, et al. Colonoscopic polypectomy and long-term prevention of colorectal-cancer deaths. N Engl J Med 2012;366:687-96.

59. Winter JM, Cameron JL, Campbell KA, et al. 1423 pancreaticoduodenectomies for pancreatic cancer: A single-institution experience. J Gastrointest Surg 2006;10:1199-210; discussion 210-1.

60. Schnelldorfer T, Ware AL, Sarr MG, et al. Long-term survival after pancreatoduodenectomy for pancreatic adenocarcinoma: is cure possible? Ann Surg 2008;247:456-62.

61. Fatima J, Schnelldorfer T, Barton J, et al. Pancreatoduodenectomy for ducta adenocarcinoma: implications of positive margin on survival. Arch Surg 2010;145:167-72

62. Laupacis A, Sackett DL, Roberts RS. An assessment of clinically useful measures of the consequences of treatment. N Engl J Med 1988;318:1728-33.

63. Sellner F, Thalhammer S, Stattner $\mathrm{S}$, et al. TNM stage and grade in predicting the prognosis of operated, non-functioning neuroendocrine carcinoma of the pancreas-a single-institution experience. J Surg Oncol 2011;104:17-21.

64. Brat DJ, Lillemoe KD, Yeo CJ, et al. Progression of pancreatic intraductal neoplasias to infiltrating adenocarcinoma of the pancreas. Am J Surg Pathol 1998:22:163-9.

65. Hruban RH, Adsay NV, Albores-Saavedra J, et al. Pancreatic intraepithelial neoplasia: a new nomenclature and classification system for pancreatic duct lesions Am J Surg Pathol 2001;25:579-86.

66. Luttges J, Zamboni G, Longnecker D, et al. The immunohistochemical mucin expression pattern distinguishes different types of intraductal papillary mucinous 
neoplasms of the pancreas and determines their relationship to mucinous noncystic carcinoma and ductal adenocarcinoma. Am J Surg Pathol 2001;25:942-8.

67. Meckler KA, Brentnall TA, Haggitt RC, et al. Familial fibrocystic pancreatic atrophy with endocrine cell hyperplasia and pancreatic carcinoma. Am J Surg Pathol 2001;25:1047-53.

68. Shi C, Klein AP, Goggins M, et al. Increased Prevalence of Precursor Lesions in Familial Pancreatic Cancer Patients. Clin Cancer Res 2009;15:7737-43.

69. Terhune PG, Phifer DM, Tosteson TD, et al. K-ras mutation in focal proliferative lesions of human pancreas. Cancer Epidemiol Biomarkers Prev 1998;7 515-21.

70. Yachida S, Jones S, Bozic I, et al. Distant metastasis occurs late during the genetic evolution of pancreatic cancer. Nature 2010;467:1114-17.

71. Wu J, Matthaei H, Maitra A, et al. Recurrent GNAS mutations define an unexpected pathway for pancreatic cyst development. Sci Trans/ Med 2011;3:92ra66.
72. Matthaei H, Schulick RD, Hruban RH, et al. Cystic precursors to invasive pancreatic cancer. Nat Rev Gastroenterol Hepatol 2011;8:141-50.

73. Sato N, Rosty C, Jansen M, et al. STK11/LKB1 Peutz-Jeghers gene inactivation in intraductal papillary-mucinous neoplasms of the pancreas. Am J Pathol 2001;159:2017-22.

74. Sohn TA, Yeo CJ, Cameron JL, et al. Intraductal papillary mucinous neoplasms of the pancreas: an updated experience. Ann Surg 2004;239:788-97; discussion 97-9.

75. Sawai $\mathbf{Y}$, Yamao K, Bhatia $\mathrm{V}$, et al. Development of pancreatic cancers during long-term follow-up of side-branch intraductal papillary mucinous neoplasms. Endoscopy 2010;42:1077-84.

76. Uehara H, Nakaizumi A, Ishikawa 0 , et al. Development of ductal carcinoma of the pancreas during follow-up of branch duct intraductal papillary mucinous neoplasm of the pancreas. Gut 2008;57:1561-5. 


\section{Correction}

Canto MI, Harinck F, Hruban RH, et al. International Cancer of the Pancreas Screening (CAPS) Consortium summit on the management of patients with increased risk for familial pancreatic cancer. Gut 2013;62:339-347.

The Collaborator Pascal Hamell's name should read Pascal Hammell.

Gut 2014;63:178. doi:10.1136/gutjnl-2012-303108corr1 


\section{Correction}

Canto MI, Harinck F, Hruban RH, et al. International Cancer of the Pancreas Screening (CAPS) Consortium summit on the management of patients with increased risk for familial pancreatic cancer. Gut 2013;62:339-47.

The Collaborator Pascal Hammell's name should read Pascal Hammel.

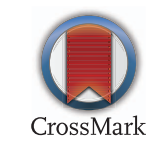

Gut 2014;63:1978. doi:10.1136/gutinl-2012-303108corr2 